\title{
Linearized $\mathrm{KdV}$ equation on a metric graph
}

\author{
Z. A. Sobirov ${ }^{1,2}$, M. I. Akhmedov, O. V. Karpova ${ }^{3,4}$, B. Jabbarova ${ }^{5}$ \\ ${ }^{1}$ Faculty of Mechanics and Mathematics, National University of Uzbekistan, Vuzgorodok, \\ 100047 Tashkent, Uzbekistan \\ ${ }^{2}$ Applied Mathematics Department of Tashkent Financial Institute, \\ 100000 Tashkent, Uzbekistan \\ ${ }^{3}$ Faculty of Physics, National University of Uzbekistan, Vuzgorodok, 100047 Tashkent, \\ Uzbekistan \\ ${ }^{4}$ Turin Polytechnic University in Tashkent, Uzbekistan \\ ${ }^{5}$ Urganch State University, Urganch, Uzbekistan \\ sobirovzar@gmail.com, ola_july@mail.ru
}

PACS 02.30.Ik, 05.45.Yv

DOI 10.17586/2220-8054-2015-6-6-757-761

We address a linearized $\mathrm{KdV}$ equation on metric star graphs with one incoming finite bond and two outgoing semi-infinite bonds. Using the theory of potentials, we reduce the problem to systems of linear integral equations and show that they are uniquely solvable under conditions of the uniqueness theorem.

Keywords: KdV, IBVP, PDE on metric graphs, exact solution, third order differential equations.

Received: 1 November 2015

\section{Introduction}

The Korteweg - de Vries (KdV) equation is of importance for many problems of physics and related fields. In particular, soliton solutions of KdV equation have found applications in fluid mechanics [1-11]. A pioneering study of KdV equation dates back to Scott Russell, who was able to model the propagation of solitary wave on the water surface in 1834. The linearized KdV provides an asymptotic description of linear, weakly dispersive long waves, such as, e.g., shallow water waves. Earlier, it was proven that via the normal form transforms the solution of the $\mathrm{KdV}$ equation can be reduced to the solution of the linear KdV equation [12]. Namely, Belashov and Vladimirov [12] numerically investigated evolution of a single disturbance $u(0, x)=u_{0} \exp \left(-x^{2} / l^{2}\right)$ and showed that in the limit $l \rightarrow 0, u_{0} l^{2}=$ const, the solution of the KdV equation is qualitatively similar to that of the linearized $\mathrm{KdV}$ equation. The boundary value problems for $\mathrm{KdV}$ equation on half lines are considered in $[2,5,7]$.

In this paper, we address the linearized $\mathrm{KdV}$ equation on a star graph $\Gamma$ with one bounded bond and two semi-infinite bonds connected at one point, called the vertex. The bonds are denoted by $B_{j}, j=1,2,3$, the coordinate $x_{1}$ on $B_{1}$ is defined from -1 to 0 , and coordinates $x_{2}$ and $x_{3}$ on the bonds $B_{2}$ and $B_{3}$ are defined from 0 to such that on each bond the vertex corresponds to 0 . On each bond we consider the linear equation:

$$
\left(\frac{\partial}{\partial t}-\frac{\partial^{3}}{\partial x_{j}^{3}}\right) u_{j}\left(x_{j}, t\right)=f_{j}(x, t), \quad t>0, x_{j} \in B_{j}, \quad j=1,2,3 .
$$

Below, we will also use the notation $x$ instead of $x_{j}(j=1,2,3)$. We treat a boundary value problem and using the method of potentials, reduce it to a system of integral equations. The solvability of the obtained system of integral equations is proven. 


\section{Formulation of the problems}

To solve the linear KdV equation on an interval, one needs to impose three boundary conditions (BC): two on the left end of thex-interval and one on the right end, (see, e.g., [5-6] and references therein). For the above star graph, we need to impose 5 BCs at the vertex point, which should provide also connection between the bonds and 2 BCs at the left side of $B_{1}$. In detail, we require:

$$
\begin{gathered}
u_{1}(-1 ; t)=\phi_{0}(t), \quad u_{1 x}(-1 ; t)=\phi_{1}(t), \\
u_{1}(0, t)=a_{2} u_{2}(0, t)=a_{3} u_{3}(0, t), \\
u_{1 x}(0 ; t)=b_{2} u_{2 x}(0 ; t)=b_{3} u_{3 x}(0 ; t), \\
u_{1 x x}(0 ; t)=a_{2}^{-1} u_{2 x x}(0 ; t)+a_{3}^{-1} u_{3 x x}(0 ; t),
\end{gathered}
$$

for $0<t<T, T=$ const.

Furthermore, we assume that the functions $f_{j}(x, t), j=1,2,3$, are smooth enough and bounded. The initial conditions are given by:

$$
u_{j}(x, 0)=0, \quad x \in \overline{B_{j}},(j=1,2,3) .
$$

It should be noted that the above vertex conditions are not the only possible ones. The main motivation for our choice is caused by the fact that they guarantee uniqueness of the solution and, if the solutions decay (to zero) at infinity, the norm (energy) conservation.

\section{Existence and uniqueness of solutions}

Lemma 1. Let $\frac{1}{b_{2}^{2}}+\frac{1}{b_{3}^{2}} \leqslant 1$. Then the problem (1)-(6) has at most one solution.

Proof of Lemma 1. Using the equation (1) one can easily get:

$$
\frac{d}{d t} \int_{a}^{b} u_{j}^{2}(x, t) d x=\left.\left(2 u_{j} u_{j x x}-u_{j x}^{2}\right)\right|_{x=a} ^{x=b}+2 \int_{a}^{b} f_{j}(x, t) u_{j}(x, t) d x
$$

for appropriate values of constants $a$ and bon each bond. We put $\phi_{0}(t) \equiv 0$. Then, the above equalities and vertex conditions (2)-(5) yield:

$$
\begin{gathered}
\frac{d}{d t}\left(e^{-\varepsilon t}\|u\|^{2}\right) \leqslant e^{-\varepsilon t}\left(\frac{1}{\varepsilon^{2}}\|f\|^{2}+\phi_{1}^{2}(t)\right), \\
\|u\|^{2} \leqslant \int_{0}^{t} e^{-\varepsilon(t-\tau)}\left(\frac{1}{\varepsilon^{2}}\|f(\cdot, \tau)\|^{2}+\phi_{1}^{2}(\tau)\right) d \tau,
\end{gathered}
$$

where $(u, v)=\int_{-1}^{0} u_{1} v_{1} d x_{1}+\int_{0}^{+\infty} u_{2} v_{2} d x_{2}+\int_{0}^{+\infty} u_{3} v_{3} d x_{3},\|u\|=\sqrt{(u, u)}$ are $L_{2}$ scalar product and norm defined on graph, $\varepsilon$ is an arbitrary positive number.

Uniqueness of the solution follows from $(7)$.

Theorem 1. Let $a_{2}^{2}+a_{3}^{2}+a_{2}^{2} a_{3}^{2}+\frac{a_{2}}{b_{3}}+\frac{a_{3}}{b_{2}} \neq 0, \frac{1}{b_{2}^{2}}+\frac{1}{b_{3}^{2}} \leqslant 1, \phi_{0}(t) \in C^{2}[0, T], \phi_{1}(t) \in$ $C^{1}[0, T]$. Then the problem (1) - (6) has a unique solution in $C^{1}\left([0, T], C^{3}(\Gamma)\right)$.

Proof of Theorem 1.

To prove the theorem, we use the following functions are called fundamental solutions of the equation $u_{t}-u_{x x x}=0$ (see $\left.[1,3,5,12,16]\right)$ :

$$
U(x, t ; \xi, \eta)= \begin{cases}\frac{1}{(t-\eta)^{1 / 3}} f\left(\frac{x-\xi}{(t-\eta)^{1 / 3}}\right), & t>\eta \\ 0 & t \leqslant \eta\end{cases}
$$




$$
V(x, t ; \xi, \eta)= \begin{cases}\frac{1}{(t-\eta)^{1 / 3}} \varphi\left(\frac{x-\xi}{(t-\eta)^{1 / 3}}\right), & t>\eta, \\ 0 & t \leqslant \eta,\end{cases}
$$

where $f(x)=\frac{\pi}{3^{1 / 3}} A i\left(-\frac{x}{3^{1 / 3}}\right), \varphi(x)=\frac{\pi}{3^{1 / 3}} B i\left(-\frac{x}{3^{1 / 3}}\right)$ for $x \geqslant 0, \varphi(x)=0$ for $x<0$ and $A i(x)$ and $B i(x)$ are the Airy functions. The functions $f(x)$ and $\varphi(x)$ are integrable and $\int_{-\infty}^{0} f(x) d x=\frac{\pi}{3}, \quad \int_{0}^{+\infty} f(x) d x=\frac{2 \pi}{3}, \quad \int_{0}^{+\infty} \varphi(x) d x=0$.

Below, we also use fractional integrals [9]:

$$
J_{(0, t)}^{\alpha} f(t):=\frac{1}{\Gamma(\alpha)} \int_{0}^{t}(t-\tau)^{\alpha-1} f(\tau) d \tau, \quad 0<\alpha<1,
$$

and the inverse of this operator, i.e. the Riemann-Liouville fractional derivatives [8, 9] defined by:

$$
D_{(0, t)}^{\alpha} f(t):=\frac{1}{\Gamma(1-\alpha)} \frac{d}{d t} \int_{0}^{t}(t-\tau)^{-\alpha} f(\tau) d \tau, \quad 0<\alpha<1 .
$$

We look for solution in the form:

$$
\begin{aligned}
u_{1}(x, t)=\int_{0}^{t} U(x, t ; 0, \eta) \varphi_{1}(\eta) d \eta+\int_{0}^{t} U(x, t ;-1, \eta) \alpha(\eta) d \eta+\int_{0}^{t} V(x, t ;-1, \eta) \beta(\eta) d \eta+F_{1}(x, t), \\
u_{2}(x, t)=\int_{0}^{t} U(x, t ; 0, \eta) \varphi_{2}(\eta) d \eta+\int_{0}^{t} V(x, t ; 0, \eta) \psi_{2}(\eta) d \eta+F_{2}(x, t), \\
u_{3}(x, t)=\int_{0}^{t} U(x, t ; 0, \eta) \varphi_{3}(\eta) d \eta+\int_{0}^{t} V(x, t ; 0, \eta) \psi_{3}(\eta) d \eta+F_{3}(x, t),
\end{aligned}
$$

where $F_{k}(x, t)=\frac{1}{\pi} \int_{0}^{t} \int_{B_{k}} U(x, t ; \xi, \eta) f_{k}(\xi, \eta) d \xi d \eta, \quad k=1,2,3$.

Satisfying the conditions (2) - (4), we have:

$$
\begin{aligned}
& f(0) \alpha(t)+\varphi(0) \beta(t)+D_{(0, t)}^{2 / 3} \int_{0}^{t} \varphi_{1}(\eta) f\left(-\frac{1}{(t-\eta)^{1 / 3}}\right) d t=\frac{1}{\Gamma\left(\frac{1}{3}\right)} D_{(0, t)}^{2 / 3}\left[\phi_{0}(t)-F_{1}(0, t)\right] \\
& f^{\prime}(0) \alpha(t)+\varphi^{\prime}(0) \beta(t)+D_{(0, t)}^{1 / 3} \int_{0}^{t} \varphi_{1}(\eta) f^{\prime}\left(-\frac{1}{(t-\eta)^{1 / 3}}\right) d t=\frac{1}{\Gamma\left(\frac{2}{3}\right)} D_{(0, t)}^{1 / 3}\left[\phi_{1}(t)-F_{1 x}(0, t)\right] \\
& f(0) \varphi_{1}(t)-a_{2} f(0) \varphi_{2}(t)-a_{2} \varphi(0) \psi_{2}(t)+\int_{0}^{t} K_{1} \alpha(\eta) d \eta+\int_{0}^{t} K_{2} \beta(\eta) d \eta= \\
&=\frac{1}{\Gamma\left(\frac{1}{3}\right)} D_{(0, t)}^{2 / 3}\left[F_{2}(0, t)-F_{1}(0, t)\right], \\
& f(0) \varphi_{1}(t)-a_{3} f(0) \varphi_{3}(t)-a_{3} \varphi(0) \psi_{3}(t)+\int_{0}^{t} K_{1} \alpha(\eta) d \eta+\int_{0}^{t} K_{2} \beta(\eta) d \eta= \\
&=\frac{1}{\Gamma\left(\frac{1}{3}\right)} D_{(0, t)}^{2 / 3}\left[F_{3}(0, t)-F_{1}(0, t)\right] .
\end{aligned}
$$


We take derivatives from $(10)-(11)$ to obtain:

$$
\begin{gathered}
f(0) \alpha^{\prime}(t)+\varphi(0) \beta^{\prime}(t)+\frac{1}{\Gamma\left(\frac{1}{3}\right)} \int_{0}^{t} K_{3} \varphi_{1}(\eta) d \eta=\frac{1}{\Gamma\left(\frac{1}{3}\right)} D_{(0, t)}^{2 / 3} \frac{d}{d t}\left[\phi_{0}(t)-F_{1}(0, t)\right], \\
f^{\prime}(0) \alpha^{\prime}(t)+\varphi^{\prime}(0) \beta^{\prime}(t)+\frac{1}{\Gamma\left(\frac{2}{3}\right)} \int_{0}^{t} K_{4} \varphi_{1}(\eta) d \eta=\frac{1}{\Gamma\left(\frac{2}{3}\right)} D_{(0, t)}^{1 / 3} \frac{d}{d t}\left[\phi_{1}(t)-F_{1 x}(0, t)\right] .
\end{gathered}
$$

From conditions (4) and (5), it follows:

$$
\begin{aligned}
& f^{\prime}(0) \varphi_{1}(t)+b_{2} f^{\prime}(0) \varphi_{2}(t)-b_{2} \varphi^{\prime}(0) \psi_{2}(t)+\frac{1}{\Gamma\left(\frac{2}{3}\right)} \int_{0}^{t} K_{5} \alpha(\eta) d \eta+\frac{1}{\Gamma\left(\frac{2}{3}\right)} \int_{0}^{t} K_{6} \beta(\eta) d \eta= \\
& =\frac{1}{\Gamma\left(\frac{2}{3}\right)} D_{(0, t)}^{1 / 3}\left[b_{2} F_{2 x}(0, t)-F_{1 x}(0, t)\right], \\
& f^{\prime}(0) \varphi_{1}(t)+b_{3} f^{\prime}(0) \varphi_{3}(t)-b_{3} \varphi^{\prime}(0) \psi_{3}(t)+\frac{1}{\Gamma\left(\frac{2}{3}\right)} \int_{0}^{t} K_{5} \alpha(\eta) d \eta+\frac{1}{\Gamma\left(\frac{2}{3}\right)} \int_{0}^{t} K_{6} \beta(\eta) d \eta= \\
& =\frac{1}{\Gamma\left(\frac{2}{3}\right)} D_{(0, t)}^{1 / 3}\left[b_{3} F_{3 x}(0, t)-F_{1 x}(0, t)\right], \\
& \quad-\frac{\pi}{3} \varphi_{1}(t)-\frac{1}{a_{2}} \frac{2 \pi}{3} \varphi_{2}(t)-\frac{1}{a_{3}} \frac{2 \pi}{3} \varphi_{3}(t)+\int_{0}^{t} K_{7} \alpha^{\prime}(\eta) d \eta+\int_{0}^{t} K_{8} \beta^{\prime}(\eta) d \eta= \\
& \quad=\frac{1}{a_{2}} F_{2 x x}(0, t)+\frac{1}{a_{3}} F_{3 x x}(0, t)-F_{1 x x}(0, t),
\end{aligned}
$$

where the kernels of integral operators defined as:

$$
\begin{aligned}
& K_{1}=\int_{\eta}^{t} \frac{1}{(t-\tau)^{2 / 3(\tau-\eta)^{1 / 3}}} f^{\prime}\left(-\frac{1}{(\tau-\eta)^{1 / 3}}\right) d \tau \\
& K_{2}=\int_{\eta}^{t} \frac{1}{(t-\tau)^{2 / 3(\tau-\eta)^{1 / 3}} \varphi^{\prime}}\left(-\frac{1}{(\tau-\eta)^{1 / 3}}\right) d \tau \\
& K_{3}=\int_{\eta}^{t} \frac{1}{(t-\tau)^{2 / 3(\tau-\eta)^{2 / 3}}} f^{\prime \prime}\left(-\frac{1}{(\tau-\eta)^{1 / 3}}\right) d \tau \text {, } \\
& K_{4}=\int_{\eta}^{t} \frac{1}{(t-\tau)^{1 / 3(\tau-\eta)^{2 / 3}}} f^{\prime \prime \prime}\left(-\frac{1}{(\tau-\eta)^{1 / 3}}\right) d \tau \text {, } \\
& K_{5}=\int_{\eta}^{t} \frac{1}{(t-\tau)^{1 / 3(\tau-\eta)^{1 / 3}}} f^{\prime \prime}\left(-\frac{1}{(\tau-\eta)^{1 / 3}}\right) d \tau \text {, } \\
& K_{6}=\int_{\eta}^{t} \frac{1}{(t-\tau)^{1 / 3(\tau-\eta)^{1 / 3}} \varphi^{\prime \prime}}\left(-\frac{1}{(\tau-\eta)^{1 / 3}}\right) d \tau \text {, } \\
& K_{7}=\int_{0}^{x} U(y+1 ; t-\eta) d y \quad, \quad K_{8}=\int_{0}^{x} V(y+1 ; t-\eta) d y \text {. }
\end{aligned}
$$


We obtained the system of integral equations (12) - (18) with respect to unknowns $\left(\alpha^{\prime}(t), \beta^{\prime}(t), \varphi_{1}(t), \varphi_{2}(t), \varphi_{3}(t), \psi_{2}(t), \psi_{3}(t)\right)$. The matrix of the coefficients $M$ of these unknowns on the off integral part of the system has a determinant:

$$
\operatorname{det} M=\frac{\pi^{4} b_{2} b_{3}}{81 a_{2} a_{3}}\left(a_{2}^{2}+a_{3}^{2}+a_{2}^{2} a_{3}^{2}+\frac{a_{2}}{b_{3}}+\frac{a_{3}}{b_{2}}\right) .
$$

Under conditions of the theorem this determinant is not singular.

According to the asymptotes of Airy functions the kernels of the integral operators are integrable (see $[14,15]$ ). Hence, it follows from the uniqueness theorem and Fredholm alternatives that the system of equations has a unique solution. Thus the solvability of the problem is proved.

\section{Acknowledgments}

This work is partly supported by a grant of the Volkswagen Foundation.

\section{References}

[1] S. Abdinazarov. The general boundary value problem for the third order equation with multiple characteristics (in Russian). Differential Equations, 1881, 3(1), P. 3-12.

[2] J.L. Bona and A.S. Fokas. Initial-boundary-value problems for linear and integrable nonlinear dispersive partial differential equations. Nonlinearity, 2008, 21, P. 195-203.

[3] L. Cattabriga. Unproblema al contorno per unaequazioneparabolica di ordinedispari. Annalidella Scuola Normale Superiore di Pisa a mat., Serie III, 13(2), 1959.

[4] J. E. Colliander, C. E. Kenig. The generalized Korteweg-de Vries equation on the half line. Commun. Partial Differ. Equations, 2002, 27(11-12), P. 2187-2266.

[5] T. D. Djuraev. Boundary value problems for mixed and mixid-composite type equations, (in Russian). Fan, Tashkent, 1979.

[6] A. V. Faminskii, N. A. Larkin. Initial-boundary value problems for quasilinear dispersive equations posed on a bounded interval. Electron. J. Differ. Equ., 2010, 2010(20).

[7] A.S. Fokas and L.Y. Sung. Initial boundary value problems for linear dispersive evolution equations on the half line. Technical report of Industrial Mathematics Institute at the University of South Carolina, 1999.

[8] M. Rahimy. Applications of fractional differential equations. Applied Mathematical Sciences, 2010, 4(50), P. 2453-2461.

[9] R.Gorenflo, F. Mainard. Fractional calculus: Integral and differential equations of fractional order. arXiv:0805.3823v1, 2008.

[10] E. Taflin. Analytic linearization of the Korteweg-De Vries equation. Pacific Journal of Mathematics, 1983, 108(1).

[11] V. Belashov, S. Vladimirov. Solitary waves in dispersive complex media: theory, simulation, application. Springer, 2005.

[12] G.B. Whitham. Linear and nonlinear waves. Pure and Applied Mathematics, Wiley-Interscience., 1974.

[13] Z.A. Sobirov, H. Uecker, M. Akhmedov. Exact solutions of the Cauchy problem for the linearized KdV equation on metric star graphs. Uz. Math. J., 2015, 3.

[14] A. R. Khashimov. Some properties of the fundamental solutions of non-stationary third order composite type equation in multidimensional domains. Journal of Nonlinear Evolution Equations and Applications, January 2013, 2013(1), P. 1-9.

[15] A.R. Khashimov, S. Yakubov. On some properties of cauchy problem for non-stationary third order composite type equation. Ufa Mathematical Journal, 2014, 6(4), P. 135-144.

[16] Z. A. Sobirov, M. I. Akhmedov, H. Uecker. Cauchy problem for the linearized KdV equation on general metric star graphs. Nanosystems: Physics, Chemistry, Mathematics, 2015, 6(1), P. 198-204. 